\title{
ULTRASONIC TESTING OF ROUGH SURFACES USING PHASED ARRAY PROBE
}

\author{
Yordan Mirchev $^{1 *}$, Mitko Minovski ${ }^{1}$ \\ AND Pavel ChuKachev ${ }^{2}$ \\ ${ }^{1}$ Institute of Mechanics, \\ Bulgarian Academy of Sciences, \\ Acad. G. Bonchev St., Bl. 4, 1113 Sofia, Bulgaria, \\ e-mail: mirchev@imbm.bas.bg; nntdd@abv.bg \\ ${ }^{2}$ Multitest Ltd, Varna 9009, Bulgaria, \\ e-mail: p.chukachev@multitest.bg
}

\begin{abstract}
The results of modeling the work of a phased array probe in a liquid medium are presented in this paper. Reflections from a smooth and periodically rough bottom surface are investigated. The amplitudes of the signals reflected by the bottom rough surface are presented. An approach to estimating the bottom rough surface by the amplitude of the reflected signal is proposed.
\end{abstract}

Keywords: phased array probe-PA, rough surface.

\section{INTRODUCTION}

In recent years, the technique with a Phased Array Probe (PA) has been used more often at ultrasonic testing of materials. It allows software control of the input ultrasonic beam axis angle, the focal length and spot width, as a result control of complex shapes in a shorter time $[1 \div 3]$.

In the present article the beam spread of a phased array transducer is represented by means of the method of modeling the propagation of ultrasonic waves in a liquid medium. It is enough widely used in solving a number of practical issues for non-destructive testing, as well as an experimental method of confirming the number of theoretical results for the calculation of the characteristics of the wave propagation in homogeneous environment $[4 \div 6]$.

At a theoretical model, the beam spread in a liquid medium is investigation, i.e. emitted pulse-signal with preset characteristics, its spread to a reflective

\footnotetext{
${ }^{*}$ Corresponding author.
}

DOI: 10.7546/EngSci.LVIII.21.01.05 
surface (discontinuity), reflection of it, and forming an information signal that carries information about the liquid medium and the discontinuity.

The purpose of the present paper is to study the spread of the ultrasonic waves emitted by the PA in liquid and their reflection (back scattering) on flat or rough surfaces.

\section{ULTRASONIC BEAM EMITTED BY THE PHASED ARRAY PROBE IN LIQUID MEDIA}

For theoretical modeling of the spread of the ultrasonic waves emitted by the PA in a liquid medium it is necessary to consider the particulars given below:

- Only longitudinal waves propagate in liquid.

- Typically, the process of signal propagation for the single-element transducer is regarded as stationary. It is assumed that the length of the pulse is realized by a sufficiently large number of oscillations. In this article the process of signal propagation is investigated as non-stationary, considering the interference signals emitted from any point of the single-element probe and signals emitted from individual elements of the PA at a preset sequence of emitted signals.

- The models are made for a pulse-echo method with bell impulse in the form of sine wave, whereby the sequence (time delays) emitted from the individual elements in the arrays is represented by the following relationship:

$$
A(r, \Delta t)=A_{0} \cdot e^{j \cdot\left[\omega_{0} \cdot\left(t-\tau_{0}\right)+\varphi\right]-\beta^{2} \cdot\left[\omega_{0} \cdot\left(t-\tau_{0}\right)+\varphi\right]^{2}},
$$

where $A_{0}$ is the maximum amplitude of the emitted pulse (in any case of the submitted work in this theoretical model the maximum value of the amplitude of the emitted pulse signal from the probe is conditionally accepted for one $\left.A_{0}=1\right), j$ - imaginary unit, $\omega_{0}=2 . \pi \cdot f_{0}$ - circular resonance frequency, $f_{0}$ - linear resonance frequency, $t$ - time of spread impulse, $\varphi_{i}=k_{i}$. $(r-H)$ phase shift between two waves, spread in one medium, $r$ and $H$-distances in which the impulses of the first and second wave propagate in the medium, respectively, from the emitted to the received impulse, $k_{i}$ - wave number of ultrasonic wave in medium with number $i, \tau_{0}=r / C_{L i}$ - time of impulse received for the first (basic) wave, corresponding to the maximum amplitude, $C_{L i}$ - values of the longitudinal ultrasonic wave propagated in the medium $i, \beta=\sqrt{\ln (2)} / \pi \cdot n_{0}$ - the coefficient of impulse shape, $n_{0}$ - the number of impulse oscillations at amplitude level $A=A_{0} / 2$. 
The values of the ultrasonic beam $P$ received by a transducer, acting on an arbitrary point in space, are written in the following form $[4 \div 6]$ :

$$
\frac{P}{P_{0}}=I . \Phi
$$

where $P_{0}$ is the pressure of emitted impulse, $I$ - the change of ultrasonic pressure of beam, spread from the probe in axial direction, $\Phi$ - the beam spread of the probe leads to the attenuation of the ultrasonic pressure, encounters the elementary point receiver, placed on the same distance from the probe depending on the spread angle between the acoustical axis and the ray toward the reflector (beam width).

In Figure 1 one of the possible focal lows of PA is schematically shown by an active group (aperture) which consists of $N a$ number of elements with length $l a$ and spacing between centers of elements $p=l a / N a$. They are arranged in a linear array with width $l p$ equal to the length of the individual element.

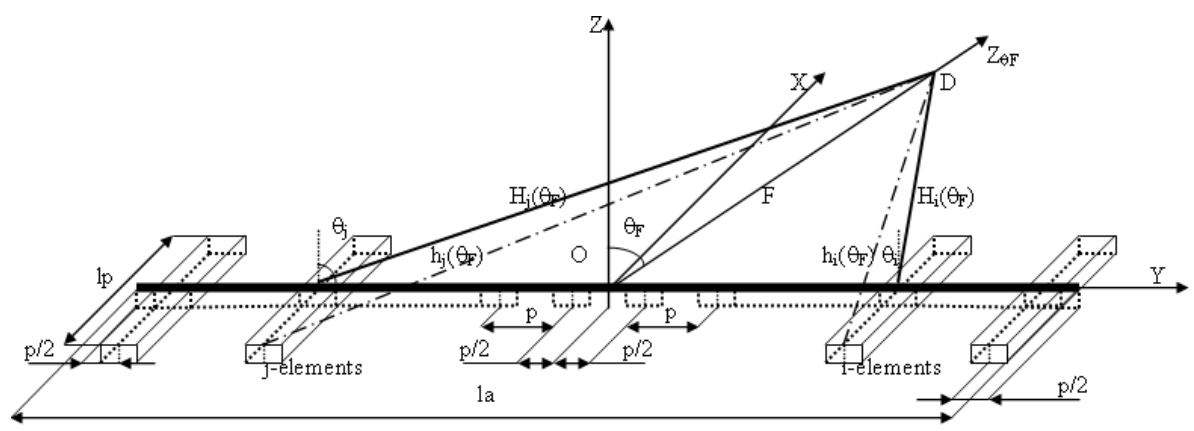

Fig. 1. Scheme of virtual aperture with individual pulsing linear array elements

The ultrasonic beam emitted from a virtual active group of PA, its spread along ultrasonic axis $I_{P A}^{N a}\left(Z_{\theta F}, \theta_{F}\right)$ and transverse to it (beam width) $\Phi_{P A}^{N a}(F, \theta)$ are calculated according to the theoretical model given in $[7,10]$ :

$$
\begin{aligned}
I_{P A}^{N a}\left(Z_{\theta F}, \theta_{F}\right) & =\frac{\max \left[\sum A\left(Z_{\theta F}, \theta_{F}\right)\right]}{\max \left[\sum A\left(F, \theta_{F}\right)\right]}, \\
\Phi_{P A}^{N a}(F, \theta) & =\frac{\max \left[\sum A(F, \theta)\right]}{\max \left[\sum A\left(F, \theta_{F}\right)\right]},
\end{aligned}
$$

where $\max \left[\sum A\left(Z_{\theta F}, \theta_{F}\right)\right]$ is the maximum of the summed amplitude emmited by each one element from active group of PA at point with distance $Z_{\theta F}$ from center $O$ of $\mathrm{PA}$ and angle $\theta_{F}$ from acoustical axis $Z, \max \left[\sum A\left(F, \theta_{F}\right)\right]$ 
is the maximum of the summed amplitude emmited by each one element from active group of $\mathrm{PA}$ at point $D$ with distance $F$ from center $O$ of $\mathrm{PA}$ and angle $\theta_{F}$ from acoustical axis $Z$, and $\max \left[\sum A(F, \theta)\right]$ is the maximum of the summed amplitude emmited by each one element from active group of PA at point $D$ with distance $F$ from center $O$ of PA and angle $\theta$ from acoustical axis $Z$.

The ultrasonic waves propagate in a liquid medium (water) with the velocity of the longitudinal ultrasonic wave $C_{w}=1.5 \mathrm{~km} / \mathrm{sec}$ and a nominal frequency $f=5 \mathrm{MHz}$. The amplitudes of the received signal are referred to the emitted signal in relative dimensionless units along the axis, as shown in Fig. 2(a). The amplitudes of the beam width are referred to the maximum amplitude of the axis beam of the calculated distance $Z$ in dimensionless units, as shown in Fig. 2(b). The distance along the ultrasonic axis $Z$ of the probe is applied in $\mathrm{mm}$ along the abscissa in Fig. 2. The beam width is calculated according to the active axis $Y$ (length $l a$ ) of the phased array probe (Fig. 1), and the length $l a$ of the rectangular one element probe from the beam spread angle $\theta$ in degrees along the abscissa in Fig. 2(b).

The ultrasonic beam emitted by an active group of PA with characteristics of each elements - length $l a=19.2 \mathrm{~mm}$, width $l p=10 \mathrm{~mm}$, number of elements $N a=32$, center-to-center distance between two successive elements (pitch) $p=l a / N a=0.6 \mathrm{~mm}$, near field length in water is $N=l a^{2} / 4 . \lambda=307 \mathrm{~mm}$, orientation of the ultrasonic beam axis to $Z$ axis at $\theta=0$ degrees, is calculated using theoretical model (3) and (4) given in $[7,10]$. The amplitude of ultrasonic beam $I$ to axis $Z$ is shown in Fig. 2(a) for unfocused phased array probe with focus length $F=10000 \mathrm{~mm}$ and for the focused PA using function Dynamic Depth Focusing (DDF) focused on each point of axis $Z$ from $0.5 \mathrm{~mm}$ to 1200 $\mathrm{mm}$. The ultrasonic beam wavefront (width) $\Phi$ shown in Fig. 2(b) is calculated with the same characteristics and the equations for unfocused phased array probe with focus length $F=10000 \mathrm{~mm}$ and the focused PA with $F=30 \mathrm{~mm}$ and $F=400 \mathrm{~mm}$.

In Figure 2(a) the gain coefficient of the amplitude:

$$
K=I_{P A}^{N a}\left(Z_{\theta F}, \theta_{F}\right) / I_{P A}^{N a}\left(Z \text { for unfocused } P A, \theta_{F}\right)
$$

is also shown for DDF PA related to unfocused PA.

The beam emitted by one rectangular element probe (conventional probe) with the size similar to those of the active group of PA is calculated using 


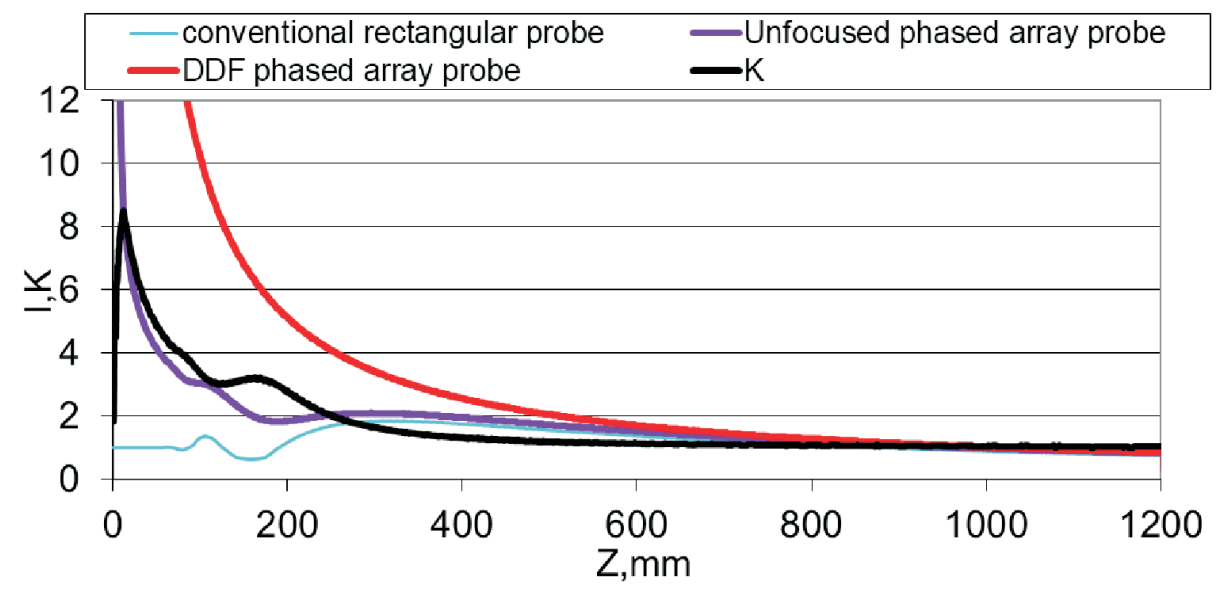

(a)

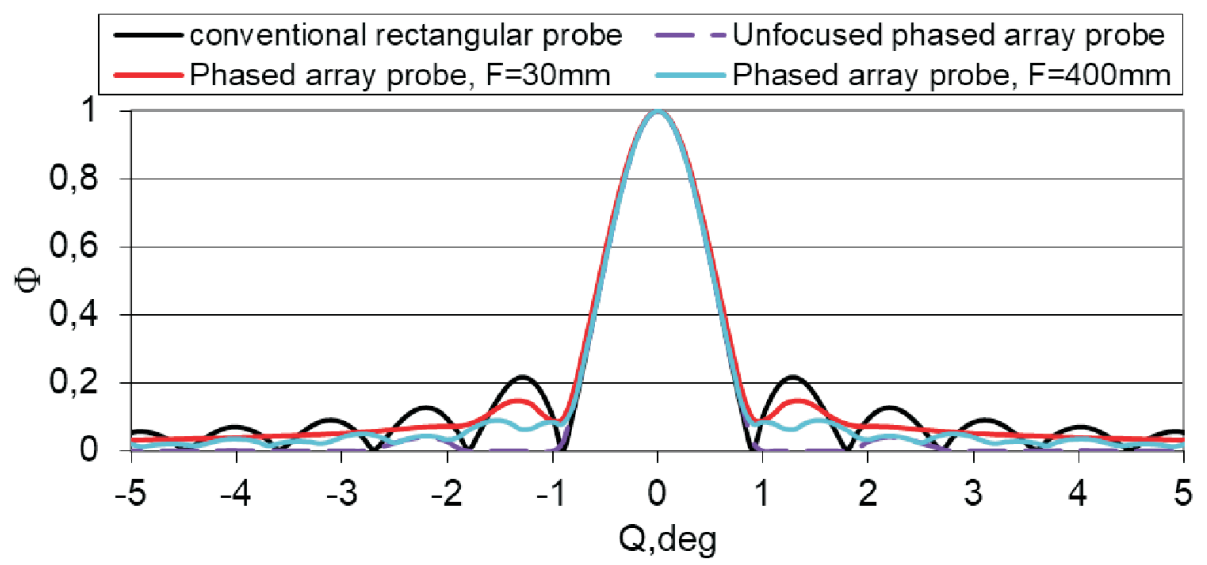

(b)

Fig. 2. Ultrasonic beam for PA and conventional rectangular single element pulse probe: (a) along the axis; (b) wavefront (width)

equations in $[4 \div 6]$ :

$$
I(Z)=\left|A(Z, t)-\frac{Z}{\sqrt{Z^{2}+m \cdot(l a / 2)^{2}}} \cdot A\left[\sqrt{Z^{2}+m \cdot(l a / 2)^{2}}, t\right]\right|,
$$




$$
\Phi(\theta)=\left|\frac{\sin \left[\frac{l a}{2} \cdot k \cdot \sin \theta\right]}{\frac{l a}{2} \cdot k \cdot \sin \theta}\right|,
$$

where $k=2 . \pi / \lambda$ is the wave number, $\lambda=C / f$ - length of wave propagated in material, $m$ - aspect ratio constant related to $l p / l a$ (when $l p / l a=0.5$ then $m \approx 1.01$ ) [1], $Z$ and $\theta$ - beam axis distance and spread angle from center of transducer element to elementary received point, respectively, placed in plane OYZ in space of ultrasonic beam.

The comparision of the results of the conventional single-element probe with PA is performed under the following conditions: the amplitude of emitted ultrasonic fields from each single-element is conditionally accepted for one, and dimensions of the linear array for PA and of the rectangular element for one crystal probe are identical.

Figure 2(a) shows that the ultrasonic beam for the unfocused PA and the conventional rectangular single-element probe are largely similar after approximately three N-near fields in the test media. Figure 2(a) also shows that the gain coefficient is $K=1$, when the ultrasonic axis distance is $Z=600 \mathrm{~mm}$ (approximately two N-near fields for water medium).

In Figure 2(b) it is shown that the ultrasonic beam wavefront is similar for PA and the rectangular single-element probe according to grating lobes. It should be noted that the similarity is valid only for the focus length $F$ of $\mathrm{PA}$, and the calculation is performed only for the long side of the rectangular element and the linear phased array $l a$.

As well known for PA the greatest distance for which can be used phased delays for focusing is the one near-field length $N$. The near-field length $N$ of $\mathrm{PA}$ is equal to the near-field length of the single rectangular element transducer under the same characteristics and acoustical environment.

We assume that to study the ultrasonic beam spread in a liquid medium for the far field of the probes, the field of PA can be replaced with some approximation from a field emitted by the one rectangular element probe.

\section{INVESTIGATED TASK}

\subsection{Back scattering echo from an infinite plane}

The back scattering echo from an infinite plane is solved by using the method of the imaginary source. Each point of the transducer element 1 can be replaced in an analogous manner to obtain an imaginary element 2

Engineering Sciences, LVIII, 2021, No. 1 
with the same emitted characteristics, located mirror-like at a distance $2 . r_{w}$ from the element 1.

In Figure 3 the ultrasonic beam which consists of emitted waves from the probe 1 , spreading at a distance $r_{w}$ in water to the axis $O_{1} Z$ is shown schematically with a solid line. The waves propagate normally to the surface of the material (steel) with acoustic impedance $Z s \approx 45$ acoustical ohms.

The ultrasonic field reflected from an infinite plane and received in the probe 1 is replaced by an imaginary source. Part of it corresponding to the normal falling down to the surface of the material is denoted by a dotted line, and another part that overlaps with the reflection of the boundary liquid-solid medium is marked with a solid line.

The echo signal from upper surface material is written in the form:

$$
\frac{P}{P_{0}}=I \cdot R_{w s} \cdot e^{-2 . \delta_{w} \cdot r_{w}},
$$

where $P_{0}$ is the pressure of emitted pulse, $P$ - the pressure of received echo, $I=I_{P A}^{N a}\left(Z_{\theta F}=2 . r_{w}, \theta_{F}=0 \mathrm{deg}\right)-$ the value of ultrasonic beam along its axis $Z$, calculated using equation (3) for PA and $I=I(Z)$ - the value of ultrasonic beam along its axis $Z$, calculated using equation (5) for conventional singleelement probe. $\delta_{w}$ is the attenuation coefficient in water, $r_{w}$ is the distance in water from the probe to the reflected material surface, $R_{w s}$ is the amplitude reflection coefficient of ultrasonic waves, which normally fall to the border of the liquid-solid body.

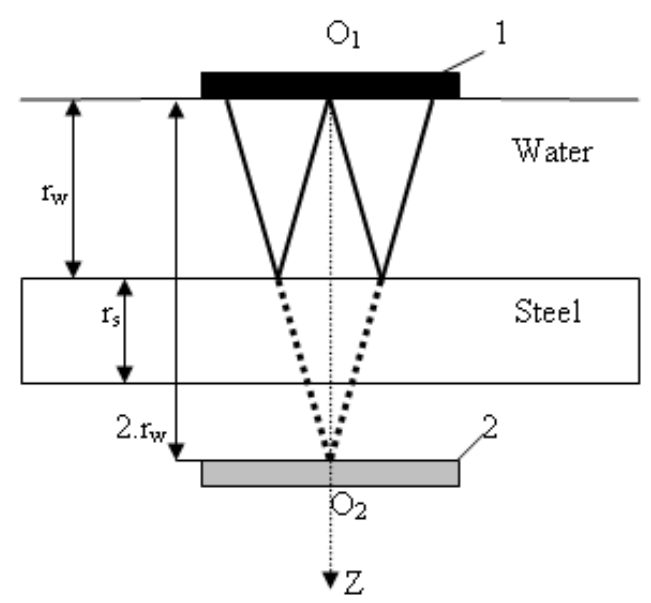

Fig. 3. Ultrasonic beam investigated with task 2.1 
The reflection coefficient $R_{w s}$ is written in the form:

$$
R_{w s}=\frac{\rho_{s} \cdot C_{s}-\rho_{w} \cdot C_{w}}{\rho_{s} \cdot C_{s}+\rho_{w} \cdot C_{w}}
$$

where $\rho_{w}$ and $\rho_{s}$ are the density of the liquid and solid body, respectively. $C_{w}$ and $C_{s}$ - the velocity of the ultrasonic wave in liquid and solid body, respectively.

\subsection{Back scattering echo from an infinite plane (steel bottom)}

When the ultrasonic field emitted by the transducer element 1 falls on the top surface of the material (steel), ultrasonic waves are partially reflected in the case 2.1, and are partially transmitted in the second environment. Transmitted ultrasonic waves, denoted by a double solid line, to the second (bottom) surface are reflected and again are transmitted to the border solid medium-liquid before falling into the transducer 1 .

The difference in the velocities of ultrasonic waves in both environments modifies the nature of the orientation of the ultrasonic field propagated in the liquid after crossing the border liquid-solid. In this case it is again appropriate to use the method of the imaginary source, as such an ultrasonic field is considered only in a solid medium, a liquid medium is replaced by a solid medium.

The replacement of the ultrasonic field, emitted by the transducer 1 in liquid with a field in a solid medium, can be represented as a field emitted by the imaginary transducer 3 denoted by a double dashed line in Fig. 4 . It is situated at a distance from the upper surface of the material equal to $n . r_{w}$, where $n=C_{w} / C_{s}$ is the ratio of the velocities of the ultrasonic wave in the liquid and solid environments. The characteristics of both the transducer 1 and 3 are similar.

The reflection from the bottom surface of the steel can be represented by a field, emitted by an imaginary transducer 4 indicated in Fig. 4 with dashed single and double lines. It is located symmetrically and mirror-like of the emitting imaginary transducer 3 to the boundary solid medium-liquid (bottom steel surface). The ultrasonic field of the imaginary transducer element 4 is denoted by the single dashed line that overlaps the material with a thick double line and double broken lines. It is analogous to the field emitted by the transducer 1 in the liquid, which is transmitted in the material and is reflected from the bottom after transmition from a solid medium in the liquid taken again from the transducer 1 .

Engineering Sciences, LVIII, 2021, No. 1 


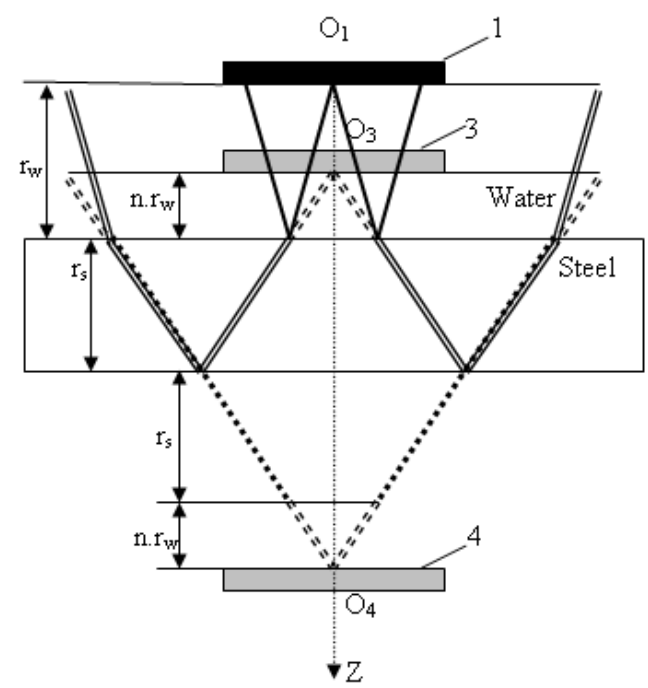

Fig. 4. Ultrasonic beam for investigated task 2.2

The back scattering signal from the bottom infinite plane is written in the form:

$$
\frac{P}{P_{0}}=I \cdot D \cdot R_{s w} \cdot e^{-2 \cdot\left(\delta_{w} \cdot r_{w}+\delta_{s} \cdot r_{s}\right)},
$$

where

$$
\begin{aligned}
& R_{s w}=\frac{\rho_{w} \cdot C_{w}-\rho_{s} \cdot C_{s}}{\rho_{w} \cdot C_{w}+\rho_{s} \cdot C_{s}}, \\
& D=\frac{4 \cdot \rho_{s} \cdot C_{s} \cdot \rho_{w} \cdot C_{w}}{\left(\rho_{s} \cdot C_{s}+\rho_{w} \cdot C_{w}\right)^{2}},
\end{aligned}
$$

$r_{s}$ is the thickness of solid material (steel), $\delta_{s}$ - the attenuation coefficient in steel, $D$ - the energy transmitting coefficient at the boundary liquid-solid body, $R_{s w}$ - the reflection amplitude coefficient at the boundary solid bodyliquid, the other characteristics as described in equations (7) and (8).

\subsection{Back scattering echo from rough surface at boundary liquid-solid body}

It is assumed that periodic roughness is formed from the same type of discontinuity, located in the test medium periodically. It changes the spread of the ultrasonic field depending on the geometrical characteristics, which modify information characteristics of the received pulse-echo in comparison with the 
same characteristics of the reflected pulse-echo from the free of discontinuity boundary medium $[8,9]$.

The ultrasonic waves emitted by the element of the transducer 1 propagate normally in the liquid to roughness, the same as in Fig. 3. The ultrasonic waves reflected from roughness are diffracted and change the ultrasonic field spreading, marked with a solid line in Fig. 5. As a result, a smaller part of it falls back into the transducer 1 and changes the characteristics of the received signal compared to the same from the smooth surface.

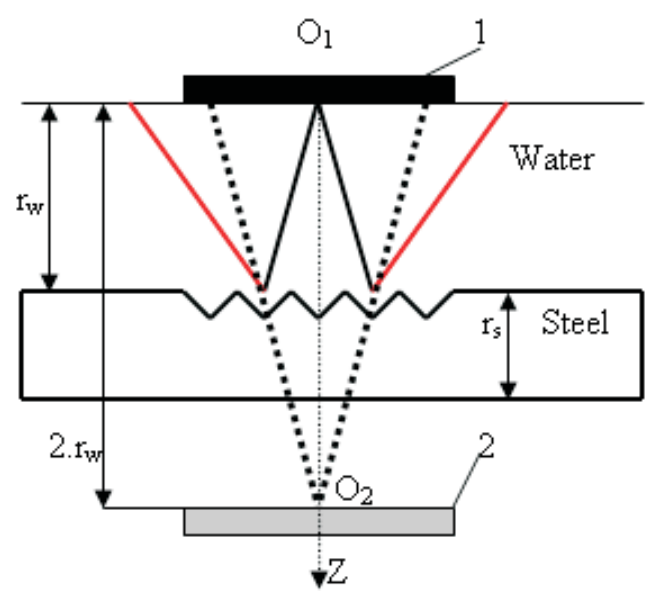

Fig. 5. Ultrasonic beam investigated with task 2.3

The changes of the pulse-echo amplitude from an rough surface compared to a smooth surface can be represented by the amplitude reflection coefficient $R u_{w s}$. In $[8,9]$ it is shown a possibility of using the values of this coefficient $R u_{w s}$ for evaluation of the geometric characteristics of the reflective rough surface. The coefficient is defined using two theoretical approaches:

- The modification method of small perturbation and the Kirchhoff approximation model are used for rough surface with RMS height $R a<\lambda / 16$ and surface correlation length $\Lambda<\lambda / 16$, where $\lambda$ is wave length. In the classical theory the back scattering signal from rough surface is not sensitive to the correlation length $\Lambda$. In [9], the modified model allows the change of correlation length $\Lambda$ to be included into the equation. This gives the possibility to see the influence from $\Lambda$ to the amplitude of reflected signal.

- The Huygens-Fresnel principle of diffraction is used for development of Kirchhoff approximation model in Geometrical Theory of Diffraction for roughness with characteristics $R a>\lambda / 16$ and $\Lambda>\lambda / 16$. 
Estimations for the case in Fig. 5 are obtained as dependence (7) multiplied by the reflection coefficient $R u_{w s}$ from the rough surface.

\subsection{Back scattering echo from rough surface at boundary solid body-liquid (steel bottom)}

The task for back scattering echo from rough surface at boundary solid body-liquid (steel bottom) is solved by means of dependence (9) multiplied by a reflection coefficient of the rough surface $R u_{s w}$ at the solid-liquid boundary. The schematic change of the spread ultrasonic field reflected from roughness in this case, again using the method of imaginary source, is shown in Fig. 6.

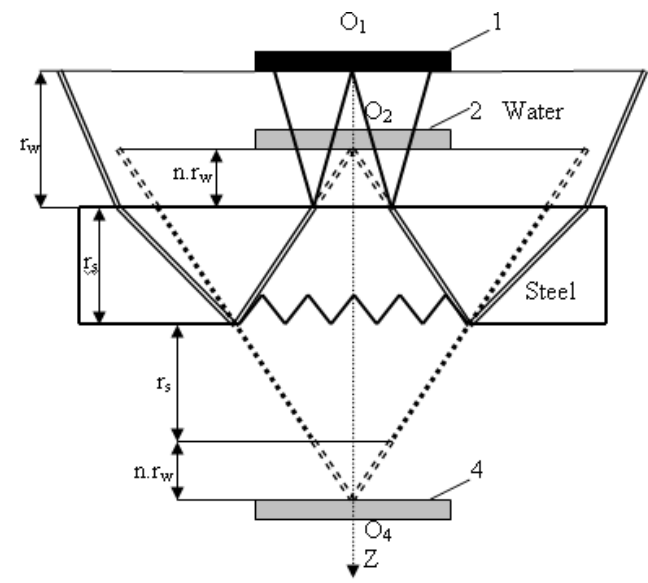

Fig. 6. Ultrasonic beam for investigated task 2.4

\section{EXPERIMENTAL INVESTIGATION}

\subsection{Experimental samples}

Ultrasonic investigations have been carried out on prepared Comparative Samples (CSs) 4 and 6 of mild steel. There are rough areas with triangular notches on one of their surfaces. The notches are done by a sharpened cutter to a preset angle. The characteristics of the CSs are given in Table 1. The surface of the CS with $R t=0 \mathrm{~mm}$ has a roughness $R a \approx 6 \mu \mathrm{m}$. 
Table 1. Characteristics of comparative samples

\begin{tabular}{|c|c|c|c|c|}
\hline CS & $\begin{array}{c}\text { Dimensions } \\
{[\mathrm{mm}]}\end{array}$ & $\begin{array}{c}\text { Apex angle of } \\
\text { roughness, } \gamma\left[{ }^{\circ}\right]\end{array}$ & $\begin{array}{c}\text { Height of roughness, } \\
R t[\mathrm{~mm}]\end{array}$ & $\begin{array}{c}\text { Size of rough } \\
\text { area }[\mathrm{mm}]\end{array}$ \\
\hline 4 & $165 \times 88 \times 38.8$ & 120 & $0.2 ; 0.4 ; 0.6 ; 0.8 ; 1.0 ; 1.2$ & $38 \times 38$ \\
\hline 6 & $165 \times 88 \times 38.6$ & 60 & $0.2 ; 0.4 ; 0.6 ; 0.8 ; 1.0$ & $38 \times 38$ \\
\hline
\end{tabular}

\subsection{Ultrasonic equipment and technique for testing}

The ultrasonic testing is carried out in immersion mode with ultrasonic equipment OmniScan MX2 and PA 5L64I1 with linear electronic scanning. The incidence angle of the longitudinal ultrasonic wave in water is 0 degrees. Phase shift of the arrays elements is used to focus the system over roughness on the upper and the bottom surfaces of the samples, respectively. The number of elements in the active group is $N a=32$, the step of electronic linear scanning is $0.6 \mathrm{~mm}$ equal to the center-to-center distance between two successive elements (pitch) $p$. The size of the active axis is $l a=19.2 \mathrm{~mm}$ (corresponding to 32 number of elements at distance $p=0.6 \mathrm{~mm}$ each) and of the passive axis is $l p=10 \mathrm{~mm}$ (length of elements in $\mathrm{PA}$ ). The position of $\mathrm{PA}$ is recorded using an encoder, according to the reference point in order to mark on the comparative sample.

The scheme of testing for task 3.1 and 3.3 is shown in Fig. 7. The ultrasonic system includes: 1 - PA 5L64I1, 2 - encoder, 3 - immersion container with distilled water, 4 - comparative sample, 5 - reflector type triangular notch. The ultrasonic equipment is denoted by 6 .

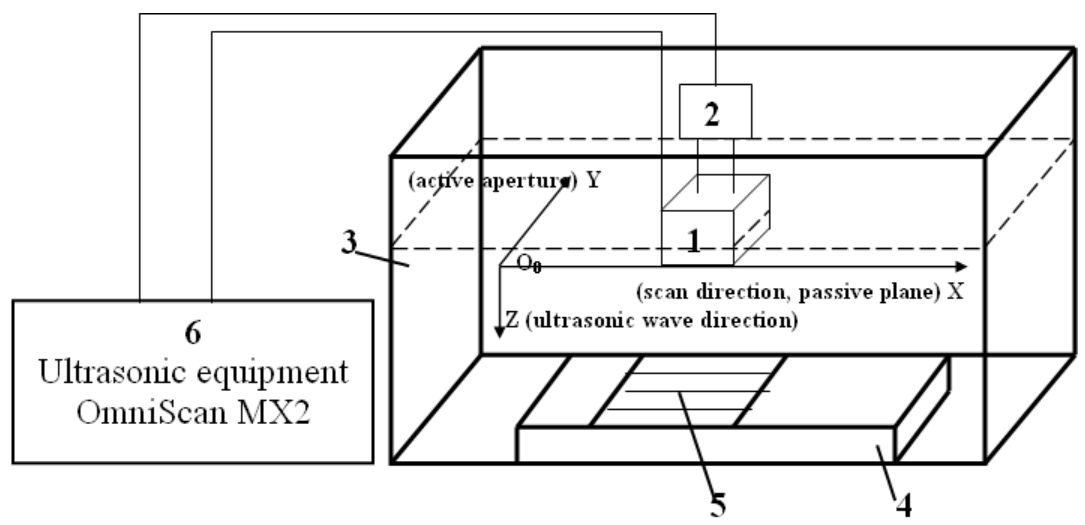

Fig. 7. Scheme of semi-automatic ultrasonic immersion testing with phased array probe 
The schemes for task 3.2 and 3.4 are identical, as the top surface of CS is flat. The roughness identified with 5 is on the bottom material surface.

One encoder is used to record the position of the moving PA to axis $X$, as active $Y$-axis and $Z$-axis have no mechanical moving of probe. The size of electronic scanning $Y$-axis is $19.8 \mathrm{~mm}$ for $0.6 \mathrm{~mm}$ pitch (corresponding to 33 focal lows with the same characteristics of focusing). Both CSs are placed successively on the $X$-axis, as the beginning of CS 4 matches with the reference point $O_{0}$. The length of the scanning $X$-axis is $360 \mathrm{~mm}$ with step $1 \mathrm{~mm}$. The distance of the forehead probe to the upper surface of the sample to $Z$-axis is $112 \mathrm{~mm}$. Information signals are recorded in digital form and can be represented as $\mathrm{A}, \mathrm{B}$ and $\mathrm{C}$ scan.

\subsection{Experimental results}

Many A-scans are recorded as the probe is moved electronically to the passive $X$-axis with step $1 \mathrm{~mm}$ and to the active $Y$-axis with step $0.6 \mathrm{~mm}$ from reference point $O_{0}$. The typical amplitude C-scans (top view) for CSs 4 and 6 are recorded and shown in Fig. 8 in color code. The shown C-scans are for the task 3.4, when the roughness is on the bottom steel surface.

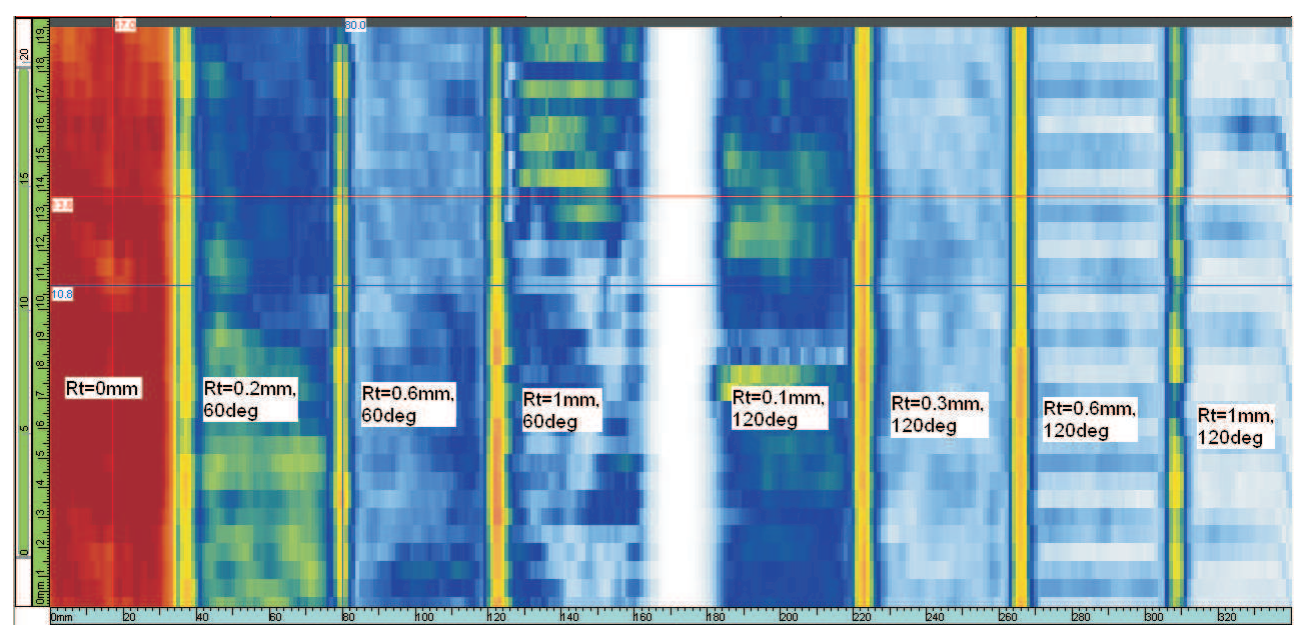

Fig. 8. C-scan of CS 4 and CS 6 for reflection from bottom roughness surfaces in immersion mode

From C-scans shown in Fig. 8, with software TomoVIEWER 2.7 R4, the amplitudes of the back scattering echo from a rough surface related to the back scattering echo from a flat surface are investigated. 
The results from the investigation of echo amplitudes from the upper rough surface are presented in Fig. 9 for wave length $\lambda=0.3 \mathrm{~mm}$ with PA-5L64I1 $(60 \mathrm{deg})$ and PA-5L64I1 (120 deg) for apex angle of roughness $\gamma=60$ degrees and $\gamma=120$ degrees, respectively. The results of echo amplitudes from the bottom rough surface are indicated with PA-5L64I1 (60 deg)-b and PA-5L64I1 (120 deg)-b for wave length $\lambda=1.18 \mathrm{~mm}$.

The results of echo amplitudes from the bottom rough steel surface for wave length $\lambda=1.47 \mathrm{~mm}$ are shown in Fig. 9 also, done in contact mode by ultrasonic equipment EPOCH 600 and conventional one crystal normal beam probe $\mathrm{K} 4 \mathrm{~N}$ with $f=4 \mathrm{MHz}$ and diameter of element $10 \mathrm{~mm}$. The results are shown with NP-K4N (60 deg)-b and NP-K4N (120 deg)-b for apex angle of roughness $\gamma=60$ degrees and $\gamma=120$ degrees, respectively [9].

The ordinate indicates the echo amplitudes $A$ in $\mathrm{dB}$, and the abscissa is a ratio of the roughness height $R t$ to the ultrasonic wave length $\lambda$ in dimensionless units.

In Figure 9 the echo amplitude dependence from the rough surfice related to the smooth surface is observed repeatedly at testing with a single element probe and PA [9]. It is assumed that small differences in dependencies are as a result of different forms of ultrasonic transducers.

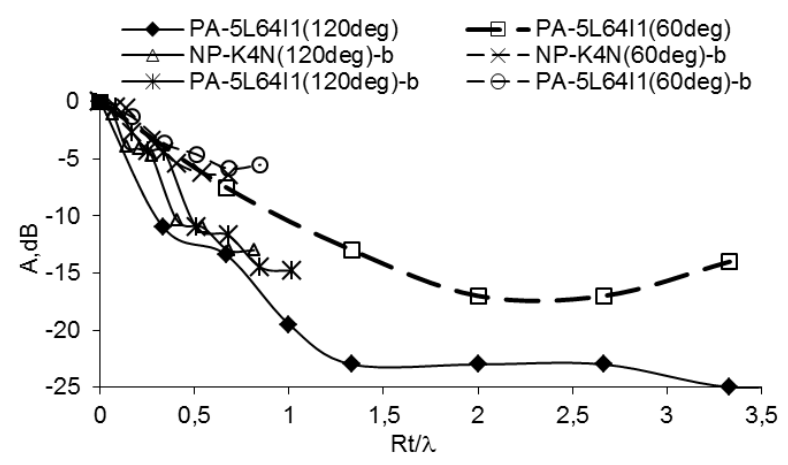

Fig. 9. Amplitudes of echoes from reflector type triangular notches with height $\mathrm{Rt}$

\section{CONCLUSION}

The ultrasonic beam for unfocused PA is approximately equal to the ultrasonic beam from a conventional single rectangular element probe in the far field for the same material. 
In the near field on the ultrasonic axis of PA the amplitude has no oscillations looks smooth and is higher according to the single rectangular element probe. This is one of the basic advantages of PA over the conventional transducer when testing is done in the near field in the material.

In the present paper a possibility of calculating the amplitude of the echo signal from a rough surface for an ultrasonic beam emitted from PA is presented by means of the theoretical approach to spreading beam in liquid and the method of the imaginary source.

The amplitude of the recevied signal is proposed as a new characteristic used to assess the surface roughness.

The nature of the echo signal amplitude dependencies from rough to smooth surface obtained with the single crystal probe $\mathrm{K} 4 \mathrm{~N}$ is confirmed by the experimental results obtained with focused PA in immersion mode.

\section{REFERENCES}

[1] A. Meindert, M. Moles, D. Kass, and T. Nelligan, Phased Array Testing - basic theory for industrial application, Canada, OLYMPUS NDT, Inc., November 2010.

[2] C. Holmes, B. W. Drinkwater, and P. D. Wilcox, Post-processing of ultrasonic phased array data for optimal performance, Insight (2005) 47 (2) 8890.

[3] M. Moles, S. LabBe, And J. Zhang, Improved focusing for thick-wall pipeline girth weld inspection using phased arrays, Insight (2005) 47 (12) 769-776.

[4] B. V. DyUdin, Immersion modelling the reflection of defects, TRTI, Taganrog (1990) (in Russian).

[5] I. N. ERmolov, Theory and Practice of the Ultrasonic Inspection, Moscow, Mashinostroenie (1981) (in Russian).

[6] I. N. Ermolov, A. Kh. Vopilkin, and V. G. Badalyan, Calculations in Ultrasonic Flaw Detection, Concise Handbook, Moscow, NPTs NK "EKhO+" (2000) (in Russian).

[7] Y. Mirchev, P. Chukachev, and M. Minovski, Examination of Reflection from a Disk Using Linear Phased Array Probe in Immersion Mode, Russian Journal of Nondestructive Testing (2017) 53 (12) 839-849, ISSN 1061-8309, (C) Pleiades Publishing, Ltd. (2017).

[8] Y. Mirchev, Modification of Rayleigh reflection from a bottom uneven surface, $11^{\text {th }}$ EC NDT, Prague 2014, paper 3.4, www.ndt.net, e-Journal \& Exhibition of Nondestructive Testing, December (2014), ISSN 1435-4934. 
[9] Y. Mirchev, S. Bukharov, V Sergienko, and I. Lvov, Characterization of damages from general corrosion of metals and alloys with ultrasonic impulse echomethod, in: Scientific Proceedings on STUME, International Conference "NDT days 2015", Sozopol, June 2015, Edition XXII, Number 2 (139), pp. 409-412, ISSN 1310-3946.

[10] V. N. Danilov and L. V. Voronkova, Basic theory and some aspects of application phased array probes, Moscow, Spectrum (2015), ISNN 978-5-44420102-2 (in Russian).

Received February 17, 2021

Engineering Sciences, LVIII, 2021, No. 1 